\title{
Sampled-data control of nonlinear systems: an overview of recent results
}

\author{
Dragan Nešić ${ }^{1}$ and Andrew R. Teel ${ }^{2}$ \\ ${ }^{1}$ Department of Electrical and Electronic Engineering, The University of \\ Melbourne, Parkville, 3010, Victoria, Australia \\ 2 CCEC, Electrical and Computer Engineering Department, \\ University of California, Santa Barbara, CA, 93106-9560
}

\begin{abstract}
Some recent results on design of controllers for nonlinear sampled-data systems are surveyed.
\end{abstract}

\section{Introduction}

The prevalence of digitally implemented controllers and the fact that the plant dynamics and/or the controller are often nonlinear strongly motivate the area of nonlinear sampled-data control systems. When designing a controller for a sampled-data nonlinear plant, one is usually faced with an important intrinsic difficulty: the exact discrete-time/sampled-data model of the plant can not be found.

The absence of a good model of a sampled-data nonlinear plant for a digital controller design has lead to several methods that use different types of approximate models for this purpose. We single out two such methods that attracted lots of attention in the sampled-data control literature and that consist of the following steps:

Method 1:

$$
\begin{gathered}
\text { continuous-time plant model } \\
\Downarrow \\
\text { continuous-time controller } \\
\Downarrow \\
\text { discretize controller } \\
\Downarrow \\
\text { implement the controller }
\end{gathered}
$$

Method 2:

$$
\begin{gathered}
\text { continuous-time plant model } \\
\Downarrow \\
\text { discretize plant model } \\
\Downarrow \\
\text { discrete-time controller } \\
\Downarrow \\
\text { implement the controller }
\end{gathered}
$$

Hence, in Method 1 (this method is often referred to as the controller emulation design) one first designs a continuous-time controller based on a continuous-time plant model. At this step the sampling is completely ignored. Then, the obtained continuous-time controller is discretized and implemented using a sampler and hold device. On the other hand, in Method 2 one first 
finds a discrete-time model of the plant, then designs a discrete-time controller based on this discrete-time model and finally implements the designed discrete-time controller using a sampler and hold device.

In principle, Method 2 is more straightforward for linear systems than for nonlinear systems. Indeed, for linear systems we can write down an explicit, exact discrete-time model while typically for nonlinear systems we cannot. Moreover, the exact discrete-time model of a linear system is linear while the exact discrete-time model for a sampled-data nonlinear system does not usually preserve important structures of the underlying continuous-time nonlinear system, like affine controls for example. Consequently, for nonlinear systems it is unusual to assume knowledge of the exact discrete-time model of the plant whereas this assumption is made in most of classical linear literature. However, often due to our inability to exactly compute the matrix exponential that generates the exact discrete-time model of a linear plant, we use its approximation and hence we actually use an approximate discrete-time plant model for controller design most of the time, even for linear systems. Consequently, we will always assume in the sequel that in Method 2 we use an approximate discrete-time model of the plant for controller design.

The main question in Method 1 is whether the desired properties of the continuous-time closed loop system that the designed controller yielded will be preserved and, if so, in what sense for the closed-loop sampled-data system. Similarly, the central question in Method 2 is whether or not the properties of the closed-loop system consisting of the exact discrete-time plant model and the discrete-time controller will have similar properties as the closedloop system consisting of the approximate discrete-time plant model and the discrete-time controller. In this paper, we present some answers to these questions for both methods. In particular, we overview several recent results on Methods 1 and 2 for general nonlinear plants that appeared in [21,27$31,41]$. These results do not contain algorithms for controller design. In the case of Method 1, controller design is the topic of the area of continuous-time nonlinear control. In the case of Method 2, controller design algorithms are yet to be developed and our results provide a unified framework for doing this.

The paper is organized as follows. In Section 2 we present results on Method 1 and in Section 3 we present results on Method 3. We do not present any proofs and the reader may refer to original references for proofs of all results.

\section{Method 1: Emulation}

Many results for nonlinear sampled-data systems uses controller emulation because of the simplicity of the method and the fact that a wide range of continuous-time controller design methods can be directly used for design of digital controllers, see $[3,33,34,42,41]$. An important drawback of the exist- 
ing nonlinear sampled-data theory is that only the questions of stability (see $[3,33,34,42])$ and input-to-state stability (see [41]) were addressed within the emulation design framework. However, one may be interested in a range of other important system theoretic properties, such as passivity and $L_{p}$ stability. It turns out that a rather general notion of dissipativity that we consider $((V, w)$-dissipativity, see Definition 1$)$ can be used to cover a range of most important system theoretic properties within a unified framework. Special cases of $(V, w)$-dissipativity are: stability, input-to-state stability, passivity, $L_{p}$ stability, forward completeness, unboundedness observability, etc. Results in this section are taken from [30] and are concerned with preservation of $(V, w)$-dissipativity under sampling in the emulation design for the cases of static state feedback controllers and open loop controls. Results on emulation of dynamic state feedback controllers can be found in [21] and they are not presented here for space reasons. Hence, results in [21,30] provide a general and unified framework for digital controller design using Method 1.

\subsection{Preliminaries}

In order to state our results precisely, we need to state some definitions and assumptions. Sets of real and natural numbers are respectively denoted as $\mathbb{R}$ and $\mathbb{N}$. The Euclidean norm of a vector $x$ is denoted as $|x|$. Given a set $B \subset \mathbb{R}^{n}$, its $\epsilon$ neighborhood is denoted as $N(B, \epsilon):=\left\{x: \inf _{z \in B}|x-z| \leq \epsilon\right\}$. A function $\gamma: \mathbb{R}_{\geq 0} \rightarrow \mathbb{R}_{\geq 0}$ is of class- $\mathcal{N}$ if it is continuous and non-decreasing. It is of class- $\mathcal{K}$ if it is continuous, zero at zero and strictly increasing; it is of class- $\mathcal{K}_{\infty}$ if it is of class- $\mathcal{K}$ and is unbounded. A continuous function $\beta: \mathbb{R}_{\geq 0} \times \mathbb{R}_{\geq 0} \rightarrow \mathbb{R}_{\geq 0}$ is of class- $\mathcal{K} \mathcal{L}$ if $\beta(\cdot, \tau)$ is of class- $\mathcal{K}$ for each $\tau \geq 0$ and $\beta(s, \cdot)$ is decreasing to zero for each $s>0$. For a given function $d(\cdot)$, we use the following notation $d\left[t_{1}, t_{2}\right]:=\left\{d(t): t \in\left[t_{1}, t_{2}\right]\right\}$. If $t_{1}=k T, t_{2}=(k+1) T$, we use the shorter notation $d[k]$, and take the norm of $d[k]$ to be the supremum of $d(\cdot)$ over $[k T,(k+1) T]$, that is

$$
\|d[k]\|_{\infty}=\operatorname{ess} \sup _{\tau \in[k T,(k+1) T]}|d(\tau)| .
$$

Consider the continuous-time nonlinear plant:

$$
\begin{aligned}
& \dot{x}=f\left(x, u, d_{c}, d_{s}\right), \\
& y=h\left(x, u, d_{c}, d_{s}\right),
\end{aligned}
$$

where $x \in \mathbb{R}^{n}, u \in \mathbb{R}^{m}$ and $y \in \mathbb{R}^{p}$ are respectively the state, control input and the output of the system and $d_{c} \in \mathbb{R}^{n_{c}}$ and $d_{s} \in \mathbb{R}^{n_{s}}$ are disturbance inputs to the system. It is assumed that $f$ and $h$ are locally Lipschitz, $f(0,0,0,0)=0$ and $h(0,0,0,0)=0$.

A starting point in the emulation design is to assume that a continuoustime (open-loop or closed loop system with an appropriately designed continuoustime controller) possesses a certain property, such as stability. We will assume 
in the sequel that the continuous system satisfies the following dissipation property:

Definition 1. The system (1), (2) is said to be $(V, w)$-dissipative if there exist a continuously differentiable function $V$, called the storage function, and a continuous function $w: \mathbb{R}^{n} \times \mathbb{R}^{m} \times \mathbb{R}^{n_{c}} \times \mathbb{R}^{n_{s}} \rightarrow \mathbb{R}$, called the dissipation rate, such that for all $x \in \mathbb{R}^{n}, u \in \mathbb{R}^{m}, d_{c} \in \mathbb{R}^{n_{c}}, d_{s} \in \mathbb{R}^{n_{s}}$ the following holds:

$$
\frac{\partial V}{\partial x} f\left(x, u, d_{c}, d_{s}\right) \leq w\left(x, u, d_{c}, d_{s}\right) .
$$

$(V, w)$-dissipation is a rather general property whose special cases are stability, input-to-state stability, passivity, etc.

Besides the continuous-time model (1), (2), we consider the exact discretetime model for (1), (2) when some of the variables in function $f$ of (1), (2) are sampled or assumed piecewise constant. More precisely, let $T>0$ be a sampling period and suppose that $u$ and $d_{s}$ in $f$ in (1) are constant during the sampling intervals, so that $u(t)=u(k T)=: u(k)$ and $d_{s}(t)=d_{s}(k T)=$ : $d_{s}(k), \forall t \in[k T,(k+1) T), \forall k \geq 0$, and $y$ is measured only at sampling instants $k T, k \geq 0$.

The exact discrete-time model for the system (1), (2) is obtained by integrating the initial value problem

$$
\dot{x}(t)=f\left(x(t), u(k), d_{c}(t), d_{s}(k)\right),
$$

with given $d_{s}(k), d_{c}[k], u(k)$ and $x_{0}=x(k)$, over the sampling interval $[k T,(k+1) T]$. Let $x(t)$ denotes the solution of the initial value problem (4) with given $d_{s}(k), d_{c}[k], u(k)$ and $x_{0}=x(k)$. Then, we can write the exact discrete-time model for (1), (2) as:

$$
\begin{aligned}
x(k+1) & =x(k)+\int_{k T}^{(k+1) T} f\left(x(\tau), u(k), d_{c}(\tau), d_{s}(k)\right) d \tau \\
& :=F_{T}^{e}\left(x(k), u(k), d_{c}[k], d_{s}(k)\right), \\
y(k) & =h\left(x(k), u(k), d_{c}(k), d_{s}(k)\right) .
\end{aligned}
$$

The sampling period $T$ is assumed to be a design parameter which can be arbitrarily assigned. In practice, the sampling period $T$ is fixed and our results could be used to determine if it is suitably small. We emphasize that $F_{T}^{e}$ in $(5)$ is not known in most cases. We denote $d_{c}:=d_{c}(0)$ and use it in the sequel.

\subsection{Main results}

We now present a series of results which provide a general framework for the emulation design method. In Theorems 1 and 2 we respectively consider the 
"weak" and the "strong" dissipation inequalities for the exact discrete-time model of the sampled-data system. Each of these dissipation inequalities is useful in certain situations, as illustrated in the last subsection, where they are applied to problems of input-to-state stability and passivity. Then several corollaries are stated for the closed-loop system consisting of (1), (2) and different kinds of static state feedback controllers. Examples are presented to illustrate different conditions in main results. For simplicity we do not present results on emulation of dynamic feedback controllers and these results can be found in [21].

Theorem 1. (Weak form of dissipation) If the system (1), (2) is (V,w)dissipative, then given any 6-tuple of strictly positive real numbers $\left(\Delta_{x}, \Delta_{u}, \Delta_{d_{c}}, \Delta_{\dot{d}_{c}}, \Delta_{d_{s}}, \nu\right)$, there exists $T^{*}>0$ such that for all $T \in\left(0, T^{*}\right)$ and all $|x| \leq \Delta_{x},|u| \leq \Delta_{u},\left|d_{s}\right| \leq \Delta_{d_{s}}$ and functions $d_{c}(t)$ that are Lipschitz and satisfy $\left\|d_{c}[0]\right\|_{\infty} \leq \Delta_{d_{c}}$ and $\left\|\dot{d}_{c}[0]\right\|_{\infty} \leq \Delta_{\dot{d}_{c}}$, the following holds for the exact discrete-time model (5) of the system (1), (2):

$$
\frac{\Delta V}{T}:=\frac{V\left(F_{T}^{e}\left(x, u, d_{c}[0], d_{s}\right)\right)-V(x)}{T} \leq w\left(x, u, d_{c}, d_{s}\right)+\nu .
$$

Under slightly stronger conditions we can prove a stronger result that is useful in some situations:

Lemma 1. If the system (1), (2) is (V,w)-dissipative, with $\frac{\partial V}{\partial x}$ being locally Lipschitz and $\frac{\partial V}{\partial x}(0)=0$, then given any quintuple of strictly positive real numbers $\left(\Delta_{x}, \Delta_{u}, \Delta_{d_{c}}, \Delta_{\dot{d}_{c}}, \Delta_{d_{s}}\right)$, there exist $T^{*}>0$ and positive constants $K_{1}, K_{2}, K_{3}, K_{4}, K_{5}$ such that for all $T \in\left(0, T^{*}\right)$ and all $|x| \leq \Delta_{x},|u| \leq \Delta_{u}$, $\left|d_{s}\right| \leq \Delta_{d_{s}}$ and functions $d_{c}(t)$ that are Lipschitz and satisfy $\left\|d_{c}[0]\right\|_{\infty} \leq \Delta_{d_{c}}$, $\left\|\dot{d}_{c}[0]\right\|_{\infty} \leq \Delta_{\dot{d}_{c}}$, we have:

$$
\begin{aligned}
& \frac{\Delta V}{T} \leq w\left(x, u, d_{c}, d_{s}\right)+ \\
& \quad T\left(K_{1}|x|^{2}+K_{2}|u|^{2}+K_{3}\left|d_{s}\right|^{2}+K_{4}\left\|d_{c}[0]\right\|_{\infty}^{2}+K_{5}\left\|\dot{d}_{c}[0]\right\|_{\infty}^{2}\right) .
\end{aligned}
$$

In the following theorem we use the strong form of dissipation inequality for the exact discrete-time model. This result is much more natural to use in the situations when the disturbances $d_{c}$ are not globally Lipschitz (see the ISS application in the next subsection and Example 1).

Theorem 2. (Strong form of dissipation) If the system (1), (2) is (V,w)dissipative, then given any quintuple of strictly positive real numbers 
$\left(\Delta_{x}, \Delta_{u}, \Delta_{d_{c}}, \Delta_{d_{s}}, \nu\right)$ there exists $T^{*}>0$ such that for all $T \in\left(0, T^{*}\right)$ and all $|x| \leq \Delta_{x},|u| \leq \Delta_{u},\left\|d_{c}[0]\right\|_{\infty} \leq \Delta_{d_{c}}$, and $\left|d_{s}\right| \leq \Delta_{d_{s}}$ the following holds for the system (5):

$$
\frac{\Delta V}{T} \leq \frac{1}{T} \int_{0}^{T} w\left(x, u, d_{c}(\tau), d_{s}\right) d \tau+\nu .
$$

It is very important to state and prove Theorems 1 and 2 for the case when a feedback controller is used to achieve $(V, w)$-dissipativity of the closed loop system. To prove result on weak dissipation inequalities, we consider the situation when static state feedback controller of the form:

$$
u=u\left(x, d_{c}, d_{s}\right)
$$

is applied to the system (1), (2). It is assumed below that the feedback (10) is bounded on compact sets. Note that this general form of feedback covers both, the full state $(u=u(x))$ and output $(u=u(y))$ static feedback. Note also, that the dissipation rate for the closed-loop system (1), (2) and (10) in the definition of $(V, w)$-dissipativity can be taken as $w=w\left(x, d_{c}, d_{s}\right)$. Direct consequences of Theorem 1 and Lemma 1 are the following corollaries:

Corollary 1. If the system (1), (2), (10) is ( $V, w)$-dissipative, then given any quintuple of strictly positive real numbers $\left(\Delta_{x}, \Delta_{d_{c}}, \Delta_{\dot{d}_{c}}, \Delta_{d_{s}}, \nu\right)$, there exists $T^{*}>0$ such that for all $T \in\left(0, T^{*}\right)$ and all $|x| \leq \Delta_{x},\left|d_{s}\right| \leq \Delta_{d_{s}}$ and all functions $d_{c}(t)$ that are Lipschitz and satisfy $\left\|d_{c}[0]\right\|_{\infty} \leq \Delta_{d_{c}},\left\|\dot{d}_{c}[0]\right\|_{\infty} \leq$ $\Delta_{\dot{d}_{c}}$, the following holds for the discrete-time model of the closed-loop system (1), (2), (10):

$$
\frac{\Delta V}{T} \leq w\left(x, d_{c}, d_{s}\right)+\nu .
$$

Corollary 2. If the system (1), (2), (10) is (V,w)-dissipative, with $\frac{\partial V}{\partial x}$ and $u\left(x, d_{c}, d_{s}\right)$ in (10) being locally Lipschitz and $\frac{\partial V}{\partial x}(0)=0$ and $u(0,0,0)=0$, then given any quadruple of strictly positive real numbers $\left(\Delta_{x}, \Delta_{d_{c}}, \Delta_{\dot{d}_{c}}, \Delta_{d_{s}}\right)$, there exists $T^{*}>0$ and positive constants $K_{1}, K_{2}, K_{3}, K_{4}$ such that for all $T \in\left(0, T^{*}\right)$ and all $|x| \leq \Delta_{x},\left|d_{s}\right| \leq \Delta_{d_{s}}$ and functions $d_{c}(t)$ that are Lipschitz and satisfy $\left\|d_{c}[0]\right\|_{\infty} \leq \Delta_{d_{c}}$ and $\left\|\dot{d}_{c}[0]\right\|_{\infty} \leq \Delta_{\dot{d}_{c}}$, the closed-loop discretetime model for system (1), (2) and (10) satisfies:

$$
\begin{aligned}
& \frac{\Delta V}{T} \leq w\left(x, d_{c}, d_{s}\right)+ \\
& \qquad\left(K_{1}|x|^{2}+K_{2}\left|d_{s}\right|^{2}+K_{3}\left\|d_{c}[0]\right\|_{\infty}^{2}+K_{4}\left\|\dot{d}_{c}[0]\right\|_{\infty}^{2}\right) .
\end{aligned}
$$


It is interesting to note that the condition on the derivative $d_{c}$ in Theorem 1 is necessary to prove the weak dissipation inequality for the discrete-time system. The following example illustrates this.

Example 1. Consider the continuous time system:

$$
\dot{x}=u(x)+d_{c}=-x+d_{c},
$$

where $x, d_{c} \in \mathbb{R}$. Using the storage function $V=\frac{1}{2} x^{2}$, the derivative of $V$ is $\dot{V}=-x^{2}+x d_{c} \leq-\frac{1}{2} x^{2}+\frac{1}{2} d_{c}^{2}$, and (12) is ISS. We will show that if $d_{c}(t)=\cos \left(\frac{t+2 T}{T}\right)$ the claim of Theorem 1 does not hold since

$$
\left\|\dot{d}_{c}\right\|_{\infty}=\left\|-\frac{1}{T} \sin \left(\frac{t+2 T}{T}\right)\right\|_{\infty}=\frac{1}{T},
$$

which goes to infinity as $T \rightarrow 0$. Assume that $u(x)$ in (12) is piecewise constant for the sampled-data system. So, the discrete-time model of the sampled-data system is

$$
x(k+1)=(1-T) x(k)+\int_{k T}^{(k+1) T} \cos \left(\frac{\tau+2 T}{T}\right) d \tau,
$$

and hence the exact discrete-time model is given by:

$$
x(k+1)=(1-T) x(k)+T[\sin (k+3)-\sin (k+2)], \quad \forall k \geq 0 .
$$

Suppose that for any given $\Delta_{x}, \Delta_{d_{c}}$ and $\nu$, there exists $T^{*}>0$ such that for all $T \in\left(0, T^{*}\right)$ and $k \geq 0$ with $|x| \leq \Delta_{x}$ and $\left\|d_{c}[0]\right\|_{\infty} \leq \Delta_{d_{c}}$ we have

$$
\frac{\Delta V}{T} \leq-\frac{1}{2} x^{2}+\frac{1}{2} d_{c}^{2}+\nu
$$

We show by contradiction that the claim is not true for our case. Direct computations yield:

$$
\begin{aligned}
\frac{\Delta V}{T} & =\frac{((1-T) x+T[\sin (3)-\sin (2)])^{2}-x^{2}}{2 T} \\
& =-x^{2}+x[\sin (3)-\sin (2)]+O(T) .
\end{aligned}
$$

Let $\tilde{x}=-0.5$, (and hence $\Delta_{x}=0.5, \Delta_{d_{c}}=1$ ). By combining (16) and (17) we conclude that there should exist $T^{*}>0$ such that $\forall T \in\left(0, T^{*}\right)$ we obtain:

$$
-\frac{1}{2} \tilde{x}^{2}+\tilde{x}[\sin (3)-\sin (2)]-\frac{1}{2} \cos (2)^{2}-\nu+O(T) \leq 0
$$

and since there exists $\nu^{*}>0$ such that $-\frac{1}{2} \tilde{x}^{2}+\tilde{x}[\sin (3)-\sin (2)]-\frac{1}{2} \cos (2)^{2}=$ $\nu^{*}$ we can rewrite $(18)$ as $\nu^{*}-\nu+O(T) \leq 0$, which is a contradiction (it does 
not hold for $\left.\nu \in\left(0, \nu^{*}\right)\right)$. Therefore, for $\Delta_{x}=0.5, \Delta_{d_{c}}=1, \nu<\nu^{*}$, there exists no $T^{*}>0$, such that $\forall T \in\left(0, T^{*}\right)$ the condition (16) holds. Note that the chosen $d_{c}(t)$ does not satisfy the condition $\left\|\dot{d}_{c}\right\|_{\infty} \leq \Delta_{\dot{d}_{c}}$ for some fixed $\Delta_{\dot{d}_{c}}>0$, which is evident from (13). Hence, in this case we can not apply Theorem 1 .

To state results on strong dissipation inequalities with static state feedback controllers, we need to consider controllers of the following form:

$$
u=u\left(x, d_{s}\right),
$$

in order to be able to state a general results on strong dissipation inequalities. This is illustrated by the following example.

Example 2. Consider the system $\dot{x}=u$, and $u=-d_{c}$, where $d_{c}(0)=0$ and $d_{c}(t)=1, \forall t>0$. Using $V(x)=x$, such that $\frac{\partial V}{\partial x}\left(-d_{c}\right)=-d_{c}$ and $w\left(x, d_{c}, d_{s}\right)=-d_{c}$. Since $u$ is sampled and $d_{c}(0)=0$, we have that $x(t)=$ $0, \forall t \in[0, T]$ and so $\Delta V / T=0$. On the other hand $\int_{0}^{T} w\left(d_{c}(\tau)\right) d \tau=-T$. Hence, if (20) was true, then we would obtain obtain $0 \leq-1+\nu$, which is not true for small $\nu$.

Corollary 3. If the system (1), (2), (19) is ( $V, w)$-dissipative, then given any quadruple of strictly positive real numbers $\left(\Delta_{x}, \Delta_{d_{c}}, \Delta_{d_{s}}, \nu\right)$, there exists $T^{*}>0$ such that for all $T \in\left(0, T^{*}\right)$ and all $|x| \leq \Delta_{x},\left\|d_{c}[0]\right\|_{\infty} \leq \Delta_{d_{c}}$, and $\left|d_{s}\right| \leq \Delta_{d_{s}}$ the following holds for the closed-loop discrete-time model of the system (1), (2) and (19):

$$
\frac{\Delta V}{T} \leq \frac{1}{T} \int_{0}^{T} w\left(x, d_{c}(\tau), d_{s}\right) d \tau+\nu .
$$

Corollary 4. If the system (1), (2), (19) is (V,w)-dissipative, with $\frac{\partial V}{\partial x}$ and $u\left(x, d_{s}\right)$ in (19) being locally Lipschitz and $\frac{\partial V}{\partial x}(0)=0, u(0,0)=0$, then given any triple of strictly positive real numbers $\left(\Delta_{x}, \Delta_{d_{c}}, \Delta_{d_{s}}\right)$, there exists $T^{*}>0$ and positive constants $K_{1}, K_{2}, K_{3}$ such that for all $T \in\left(0, T^{*}\right)$ and all $|x| \leq \Delta_{x},\left\|d_{c}[0]\right\|_{\infty} \leq \Delta_{d_{c}}$, and $\left|d_{s}\right| \leq \Delta_{d_{s}}$ the closed-loop discrete-time model for the system (1), (2) and (19) satisfies:

$$
\frac{\Delta V}{T} \leq \frac{1}{T} \int_{0}^{T} w\left(x, d_{c}(\tau), d_{s}\right) d \tau+T\left(K_{1}|x|^{2}+K_{2}\left|d_{s}\right|^{2}+K_{3}\left\|d_{c}[0]\right\|_{\infty}^{2}\right) .
$$




\subsection{Applications}

The weak and strong discrete-time dissipation inequalities in Theorems 1 and 2 are tools that can be used to show that the trajectories of the sampleddata system with an emulated controller have a certain property. In order to illustrate what kind of properties can be proved for the sampled-data system using the weak or strong inequalities, we apply our results to two important system properties: input-to-state stability and passivity. The results on input-to-state stability were proved in [30] and in [31]. The sketch of proof of the result on passivity can be found in [30]. Further applications of weak and strong inequalities to investigation of $L_{p}$ stability, integral ISS, etc. are possible and are interesting topics for further research.

Input-to-state stability: Let us suppose that the system

$$
\dot{x}(t)=\tilde{f}\left(x(t), u(t), d_{c}(t)\right)
$$

can be rendered ISS using the locally Lipschitz static state feedback

$$
u=u(x),
$$

in the following sense:

Definition 2. The system $\dot{x}=f\left(x, d_{c}\right)$ is input-to-state stable if there exists $\beta \in \mathcal{K} \mathcal{L}$ and $\gamma \in \mathcal{K}$ such that the solutions of the system satisfy $|x(t)| \leq$ $\beta(|x(0)|, t)+\gamma\left(\left\|d_{c}\right\|_{\infty}\right), \quad \forall x(0), d_{c} \in \mathcal{L}_{\infty}, \forall t \geq 0$.

Suppose also that the feedback needs to be implemented using a sampler and zero order hold, that is:

$$
u(t)=u(x(k)) \quad t \in[k T,(k+1) T), k \geq 0
$$

The following result was first proved in [41] and an alternative proof was presented in [30]. The proof in [30] is based on the result on strong dissipation inequalities given in Corollary 3 and the results in [27]. In this case, the results on weak dissipation inequalities could not be used. This is because we do not want to impose the condition that the disturbances are Lipschitz when proving the following result, and that is a standing assumption in results on weak dissipation inequalities.

Corollary 5. If the continuous time system (21), (22) is ISS, then there exist $\beta \in \mathcal{K} \mathcal{L}, \gamma \in \mathcal{K}$ such that given any triple of strictly positive numbers $\left(\Delta_{x}, \Delta_{d_{c}}, \nu\right)$, there exists $T^{*}>0$ such that for all $T \in\left(0, T^{*}\right),\left|x\left(t_{0}\right)\right| \leq \Delta_{x}$, $\left\|d_{c}\right\|_{\infty} \leq \Delta_{d_{c}}$, the solutions of the sampled-data system (21), (23) satisfy:

$$
|x(t)| \leq \beta\left(\left|x\left(t_{0}\right)\right|, t-t_{0}\right)+\gamma\left(\left\|d_{c}\right\|_{\infty}\right)+\nu,
$$

$\forall t \geq t_{0} \geq 0$. 
Corollary 5 states that if the continuous-time closed loop system is ISS, then the sampled-data system with the emulated controller will be semiglobally practically ISS, where the sampling period is the parameter that we can adjust. Besides the above given property that is presented in an $L_{\infty}$ setting, we can prove the following integral (or $L_{2}$ ) version of the same result that was proved in [31].

Corollary 6. If the system (21), (22) is ISS, then given any quadruple of strictly positive real numbers $\left(\Delta_{x}, \Delta_{w}, \nu_{1}, \nu_{2}\right)$ there exists $T^{*}>0$ such that for all $T \in\left(0, T^{*}\right),|x(0)| \leq \Delta_{x}$ and $\|w\|_{\infty} \leq \Delta_{w}$, the following inequality holds for the sampled-data system (21), (23) satisfy:

$$
\int_{0}^{t} \alpha_{3}(|x(s)|) d s \leq \alpha_{2}(|x(0)|)+\int_{0}^{t} \gamma(|w(s)|) d s+\nu_{1} t+\nu_{2},
$$

for all $t \geq 0$.

Passivity: Consider the continuous time system with outputs

$$
\dot{x}=f(x, u), \quad y=h(x, u),
$$

where $x \in \mathbb{R}^{n}, y, u \in \mathbb{R}^{m}$ and assume that the system is passive, that is $(V, w)$-dissipative, where $V: \mathbb{R}^{n} \rightarrow \mathbb{R}_{\geq 0}$ and $w=y^{T} u$. We can apply either results of Theorem 1 or 2 since $u$ is a piecewise constant input, to obtain that the discrete-time model satisfies the following: for any $\left(\Delta_{x}, \Delta_{u}, \nu\right)$ there exists $T^{*}>0$ such that for all $T \in\left(0, T^{*}\right),|x| \leq \Delta_{x},|u| \leq \Delta_{u}$ we have:

$$
\frac{\Delta V}{T} \leq y^{T} u+\nu
$$

In stability and ISS applications, adding $\nu$ in the dissipation inequality deteriorated the property, but the deterioration was gradual. However, in (27) $\nu$ acts as an infinite energy storage (finite power source) and hence it contradicts the definition of a passive system as one that can not generate power internally. As a result, conditions which guarantee that $\nu$ in (27) can be set to zero are very important. These conditions are spelled out in the next corollary:

Corollary 7. Suppose that the system (26) is strictly input and state passive in the following sense: the storage function has gradient $\frac{\partial V}{\partial x}$ that is locally Lipschitz and zero at zero and the dissipation rate can be taken as $w(x, y, u)=$ $y^{T} u-\psi_{1}(x)-\psi_{2}(u)$, where $\psi_{1}$ and $\psi_{2}$ are positive definite functions that are locally quadratic. Then given any pair of strictly positive numbers $\left(\Delta_{x}, \Delta_{u}\right)$ there exists $T^{*}>0$ such that for all $T \in\left(0, T^{*}\right),|x| \leq \Delta_{x},|u| \leq \Delta_{u}$ we have:

$$
\frac{\Delta V}{T} \leq y^{T} u-\frac{1}{2} \psi_{1}(x)-\frac{1}{2} \psi_{2}(u) .
$$




\section{Method 2: Approximate discrete-time model design}

As we already indicated in the introduction, a majority of nonlinear and linear discrete-time control literature is based on the assumption, which is unrealistic in general, that the exact discrete-time model of the plant is known. In reality, even in the case of linear systems we use an approximate discretetime model for the discrete-time controller design. We recognize this fact and whenever we refer to Method 2, we always assume that only an approximate discrete-time model is available for the controller design.

One may be tempted to believe that if a controller is designed for an approximate discrete-time model of the plant with a sufficiently small sampling period then the same controller will stabilize the exact discrete-time model. Note that if this was true then one could directly apply all the existing theory that assumes that the exact discrete-time model is known. However, this reasoning is wrong since no matter how small the sampling period is, we may always find a controller that stabilizes the approximate model for that sampling period but destabilizes the exact model for the same sampling period, as illustrated by the following example.

Example 3. Consider the triple integrator $\dot{x}_{1}=x_{2}, \dot{x}_{2}=x_{3}, \dot{x}_{3}=u$, its Euler approximate model

$$
\begin{aligned}
& x_{1}(k+1)=x_{1}(k)+T x_{2}(k) \\
& x_{2}(k+1)=x_{2}(k)+T x_{3}(k) \\
& x_{3}(k+1)=x_{3}(k)+T u(k),
\end{aligned}
$$

and a minimum-time dead-beat controller for the Euler discrete-time model given by

$$
u(k)=\left(-\frac{x_{1}(k)}{T^{3}}-\frac{3 x_{2}(k)}{T^{2}}-\frac{3 x_{3}(k)}{T}\right) .
$$

The closed loop system consisting of (29) and (30) has all poles equal to zero for all $T>0$ and hence this discrete-time Euler-based closed loop system is asymptotically stable for all $T>0$. On the other hand, the closed loop system consisting of the exact discrete-time model of the triple integrator and controller $(30)$ has a pole at $\approx-2.644$ for all $T>0$. Hence, the closedloop sampled-data control system is unstable for all $T>0$ (and, hence, also for arbitrarily small $T$ )! So we see that, to design a stabilizing controller using Method 2, it is not sufficient to design a stabilizer for an approximate discrete-time model of the plant for sufficiently small $T$. Extra conditions are needed!

Several control laws in the literature have been designed based on approximate discrete-time models of the plant, see $[8,12,23]$. These results are always concerned with a particular plant model and a particular approximate discrete-time model (usually the Euler approximation) and hence they 
are not very general. On the other hand, we present a rather general result for a large class of plants, a large class of approximate discrete-time models and the conditions we obtain are readily checkable. For different approximate discretization procedures see $[25,38,39,37]$.

Results in this section are based mainly on [28] and they address the design of stabilizing static state feedback controllers based on approximate discrete-time plant models. A more general result was recently proved in [29] where conditions are presented for dynamic feedback controllers that are designed for approximate discrete-time models of sampled-data differential inclusions to prove stability with respect to arbitrary non-compact sets.

\subsection{Main results}

Consider the nonlinear continuous-time control system:

$$
\dot{x}=f(x, u) \quad x(0)=x_{\circ}
$$

where $x \in \mathbb{R}^{n}, u \in \mathbb{R}^{m}$ are respectively the state and control vectors. The function $f$ is assumed to be such that, for each initial condition and each constant control, there exists a unique solution defined on some (perhaps bounded) interval of the form $[0, \tau)$. The control is taken to be a piecewise constant signal $u(t)=u(k T)=: u(k), \forall t \in[k T,(k+1) T[, k \in \mathbb{N}$ where $T>0$ is a sampling period. We assume that the state measurements $x(k):=$ $x(k T)$ are available at sampling instants $k T, k \in \mathbb{N}$. The sampling period $T$ is assumed to be a design parameter which can be arbitrarily assigned (in practice, the sampling period $T$ is fixed and our results could be used to determine if it is suitably small).

Suppose that we want to design a control law for the plant (31) using Method 2. The controller will be implemented digitally using a sampler and zero order hold element. As a first step in this direction we need to specify a discrete-time model of the plant (31), which describes the behavior of the system at sampling instants. We consider the difference equations corresponding to the exact plant model and its approximation respectively:

$$
\begin{aligned}
& x(k+1)=F_{T}^{e}(x(k), u(k)) \\
& x(k+1)=F_{T}^{a}(x(k), u(k))
\end{aligned}
$$

which are parameterized with the sampling period $T$. We emphasize that $F_{T}^{e}$ is not known in most cases. We will think of $F_{T}^{e}$ and $F_{T}^{a}$ as being defined globally for all small $T$ even though the initial value problem (31) may exhibit finite escape times. We do this by defining $F_{T}^{e}$ arbitrarily for pairs $(x(k), u(k))$ corresponding to finite escapes and noting that such points correspond only to points of arbitrarily large norm as $T \rightarrow 0$, at least when $f$ is locally bounded. So, the behavior of $F_{T}^{e}$ will reflect the behavior of (31) as long as $\left(x(k), u_{T}(x(k))\right)$ remains bounded with a bound that is allowed to grow as $T \rightarrow 0$. This is consistent with our main results that guarantee practical 
asymptotic stability that is semiglobal in the sampling period, i.e., as $T \rightarrow$ 0 the set of points from which convergence to an arbitrarily small ball is guaranteed to contain an arbitrarily large neighborhood of the origin.

In general, one needs to use small sampling periods $T$ since the approximate plant model is a good approximation of the exact model typically only for small $T$. It is clear then that we need to be able to obtain a controller $u_{T}(x)$ which is, in general, parameterized by $T$ and which is defined for all small $T$. For simplicity, we consider only static state feedback controllers.

For a fixed $T>0$, consider systems (32), (33) with a given controller $u(k)=u_{T}(x(k))$. We denote the state of the closed-loop system (32) (respectively (33)) with the given controller at time step $k$ that starts from $x(0)$ as $x^{e}(k, x(0))$ or $x_{k}^{e}\left(\right.$ respectively $x^{a}(k, x(0))$ or $\left.x_{k}^{a}\right)$. We introduce the error:

$$
\varepsilon_{k}(\xi, z):=x^{e}(k, \xi)-x^{a}(k, z),
$$

and also use the notation $\varepsilon_{k}(\xi):=\varepsilon_{k}(\xi, \xi)=x^{e}(k, \xi)-x^{a}(k, \xi)$. In our results (see Theorems 3 and 4 ), we will make a stability assumption on the family of closed-loop approximate plant models and will draw a conclusion about stability of the family of closed-loop exact plant models by invoking assumptions about the closeness of solutions between the two families.

One-step consistency The first type of closeness we will use is characterized in the following definition. It guarantees that the error between solutions starting from the same initial condition is small, over one step, relative to the size of the step. The terminology we use is based on that used in the numerical analysis literature (see [39]).

Definition 3. The family $\left(u_{T}, F_{T}^{a}\right)$ is said to be one-step consistent with $\left(u_{T}, F_{T}^{e}\right)$ if, for each compact set $\mathcal{X} \subset \mathbb{R}^{n}$, there exist a function $\rho \in \mathcal{K}_{\infty}$ and $T^{*}>0$ such that, for all $x \in \mathcal{X}$ and $\left.T \in\right] 0, T^{*}[$, we have

$$
\left|F_{T}^{e}\left(x, u_{T}(x)\right)-F_{T}^{a}\left(x, u_{T}(x)\right)\right| \leq T \rho(T) .
$$

A sufficient condition for one-step consistency is the following:

Lemma 2. If

1. $\left(u_{T}, F_{T}^{a}\right)$ is one-step consistent with $\left(u_{T}, F_{T}^{\text {Euler }}\right)$ where $F_{T}^{\text {Euler }}(x, u):=$ $x+T f(x, u)$,

2. for each compact set $\mathcal{X} \subset \mathbb{R}^{n}$ there exist $\rho \in \mathcal{K}_{\infty}, M>0, T^{*}>0$ such that, for all $T \in] 0, T^{*}[$ and all $x, y \in \mathcal{X}$,

(a) $\left|f\left(y, u_{T}(x)\right)\right| \leq M$,

(b) $\left|f\left(y, u_{T}(x)\right)-f\left(x, u_{T}(x)\right)\right| \leq \rho(|y-x|)$,

then $\left(u_{T}, F_{T}^{a}\right)$ is one-step consistent with $\left(u_{T}, F_{T}^{e}\right)$. 
Multi-step consistency The second type of closeness we will use is characterized in terms of the functions $F_{T}^{e}, F_{T}^{a}$ and $u_{T}(x)$ in the next definition. It will guarantee (see Lemma 3 ) that the error between solutions starting from the same initial condition is small over multiple steps corresponding "continuous-time" intervals with length of order one.

Definition 4. The family $\left(u_{T}, F_{T}^{a}\right)$ is said to be multi-step consistent with $\left(u_{T}, F_{T}^{e}\right)$ if, for each $L>0, \eta>0$ and each compact set $\mathcal{X} \subset \mathbb{R}^{n}$, there exist a function $\alpha: \mathbb{R}_{\geq 0} \times \mathbb{R}_{\geq 0} \rightarrow \mathbb{R}_{\geq 0} \cup\{\infty\}$ and $T^{*}>0$ such that, for all $\left.T \in\right] 0, T^{*}[$ we have that $\{x, z \in \mathcal{X},|x-z| \leq \delta\}$ implies

$$
\left|F_{T}^{e}\left(x, u_{T}(x)\right)-F_{T}^{a}\left(z, u_{T}(z)\right)\right| \leq \alpha(\delta, T)
$$

and

$$
k \leq L / T \quad \Longrightarrow \quad \alpha^{k}(0, T):=\overbrace{\alpha(\cdots \alpha(\alpha}^{k}(0, T), T) \cdots, T) \leq \eta .
$$

In terms of trajectory error over "continuous-time" intervals with length of order one, multi-step consistency gives the following:

Lemma 3. If $\left(u_{T}, F_{T}^{a}\right)$ is multi-step consistent with $\left(u_{T}, F_{T}^{e}\right)$ then for each compact set $\mathcal{X} \subset \mathbb{R}^{n}, L>0$ and $\eta>0$ there exists $\hat{T}>0$ such that, if $T$ and $\xi$ satisfy

$$
T \in] 0, \hat{T}\left[, \quad x_{k}^{a}(\xi) \in \mathcal{X} \quad \forall k: k T \in[0, L],\right.
$$

then

$$
\left|\varepsilon_{k}(\xi)\right| \leq \eta \quad \forall k: k T \in[0, L] .
$$

An interesting sufficient condition for multi-step consistency is given in the following:

Lemma 4. If, for each compact set $\mathcal{X} \subset \mathbb{R}^{n}$, there exist $K>0, \rho \in \mathcal{K}_{\infty}$ and $T^{*}>0$ such that for all $\left.T \in\right] 0, T^{*}[$ and all $x, z \in \mathcal{X}$ we have

$$
\left|F_{T}^{e}\left(x, u_{T}(x)\right)-F_{T}^{a}\left(z, u_{T}(z)\right)\right| \leq(1+K T)|x-z|+T \rho(T)
$$

then $\left(u_{T}, F_{T}^{a}\right)$ is multi-step consistent with $\left(u_{T}, F_{T}^{e}\right)$.

Relative to the one-step consistency condition, the condition of Lemma 4 is guaranteed by one-step consistency plus the following type of Lipschitz condition on either the family $\left(u_{T}, F_{T}^{e}\right)$ or the family $\left(u_{T}, F_{T}^{a}\right)$ :

for each compact set $\mathcal{X} \subset \mathbb{R}^{n}$ there exist $K>0$ and $T^{*}>0$ such that for all $x, z \in \mathcal{X}$ and all $T \in] 0, T^{*}[$,

$$
\left|F_{T}\left(x, u_{T}(x)\right)-F_{T}\left(z, u_{T}(z)\right)\right| \leq(1+K T)|x-z| .
$$


This condition is guaranteed for $F_{T}^{e}$ when $f(x, u)$ and $u_{T}(x)$ are locally Lipschitz (uniformly in small $T$ ). Note that no continuity assumptions on $u_{T}(x)$ were made in Lemma 2 to guarantee one-step consistency. The condition given in Lemma 4 for multi-step consistency is similar to conditions used in the numerical analysis literature (e.g., see conditions ( $i$ ) and (iii) of Assumption 6.1.2 in [39, pg.429]).

One-step and multi-step consistency do not imply each other and this is one motivation for developing different stability theorems that rely on one of these properties. Example 4 in Section 3.3 shows that multi-step consistency may not hold when one-step consistency does hold. That one-step consistency may not hold when multi-step consistency does hold can be seen from the plant $\dot{x}=x+u$ with Euler approximation $x(k+1)=x(k)+T(x(k)+u(k))=$ $F_{T}^{a}(x(k), u(k))$ and controller $u_{T}(x)=-\left(\frac{1}{T}+1\right) x$. The exact discrete-time model is $x(k+1)=e^{T} x(k)+\left(e^{T}-1\right) u(k)$ and we have $F_{T}^{a}\left(x, u_{T}(x)\right) \equiv 0$ and $F_{T}^{e}\left(x, u_{T}(x)\right)=\left(1-\frac{e^{T}-1}{T}\right) x$. Since, for $x$ in a compact set, $F_{T}^{e}\left(x, u_{T}(x)\right)$ is of order $T$ we do not have one-step consistency. On the other hand, it follows from $F_{T}^{a}\left(x, u_{T}(x)\right) \equiv 0$ and the fact that $F_{T}^{e}\left(x, u_{T}(x)\right)$ is of order $T$ that we do have multi-step consistency. Indeed, for each compact set $\mathcal{X} \subset \mathbb{R}$ and each $\eta>0$ there exist strictly positive numbers $K, T^{*}$ such that, for all $x, z \in \mathcal{X}$, $T \in] 0, T^{*}[, k \geq 0$,

$$
\begin{aligned}
\left|F_{T}^{e}\left(x, u_{T}(x)\right)-F_{T}^{a}\left(z, u_{T}(z)\right)\right| & =\left|F_{T}^{e}\left(x, u_{T}(x)\right)\right| \leq K T \\
& :=\alpha(\delta, T)=\alpha^{k}(0, T) \leq \eta .
\end{aligned}
$$

\subsection{Stability properties}

We now give conditions on the family $\left(u_{T}, F_{T}^{a}\right)$ that guarantee asymptotic stability for the family $\left(u_{T}, F_{T}^{e}\right)$. As we have already seen in Example 3, it is not enough to assume simply that each member of the family $\left(u_{T}, F_{T}^{a}\right)$ is asymptotically stable (at least for small $T$ ). Instead, we will impose partial uniformity of the stability property over all small $T$. For that, we make the following definitions:

Definition 5. Let $\beta \in \mathcal{K} \mathcal{L}$ and let $N \subset \mathbb{R}^{n}$ be an open (not necessarily bounded) set containing the origin.

1. The family $\left(u_{T}, F_{T}\right)$ is said to be $(\beta, N)$-stable if there exists $T^{*}>0$ such that for each $T \in] 0, T^{*}[$, the solutions of the system

$$
x(k+1)=F_{T}\left(x(k), u_{T}(x(k))\right)
$$

satisfy

$$
|x(k, x(0))| \leq \beta(|x(0)|, k T), \forall x(0) \in N, k \geq 0 .
$$


2. The family $\left(u_{T}, F_{T}\right)$ is said to be $(\beta, N)$-practically stable if for each $R>0$ there exists $T^{*}>0$ such that for each $\left.T \in\right] 0, T^{*}[$ the solutions of (42) satisfy:

$$
|x(k, x(0))| \leq \beta(|x(0)|, k T)+R, \forall x(0) \in N, k \geq 0 .
$$

An equivalent Lyapunov formulation of $\left(\beta, \mathbb{R}^{n}\right)$-stability is the following (local versions can also be formulated but are more tedious to state because of the need to keep track of basins of attraction):

Lemma 5. The following statements are equivalent:

1. There exists $\beta \in \mathcal{K} \mathcal{L}$ such that the family $\left(u_{T}, F_{T}\right)$ is $\left(\beta, \mathbb{R}^{n}\right)$-stable.

2. There exist $T^{*}>0, \alpha_{1}, \alpha_{2} \in \mathcal{K}_{\infty}, \alpha_{3} \in \mathcal{K}$ and for each $\left.T \in\right] 0, T^{*}\left[, V_{T}\right.$ : $\mathbb{R}^{n} \rightarrow \mathbb{R}_{\geq 0}$ such that $\left.\forall x \in \mathbb{R}^{n}, \forall T \in\right] 0, T^{*}[$ we have:

$$
\begin{array}{r}
\alpha_{1}(|x|) \leq V_{T}(x) \leq \alpha_{2}(|x|), \\
V_{T}\left(F_{T}\left(x, u_{T}(x)\right)\right)-V_{T}(x) \leq-T \alpha_{3}(|x|), .
\end{array}
$$

In our first main result (Theorem 3 ) we will show that if the family $\left(u_{T}, F_{T}^{a}\right)$ is $(\beta, N)$-stable and multi-step consistent with $\left(u_{T}, F_{T}^{e}\right)$ then the family $\left(u_{T}, F_{T}^{e}\right)$ is $(\beta, N)$-practically stable. We will also show (in Theorem 4 ) that the multi-step consistency assumption can be changed to a one-step consistency assumption when $(\beta, N)$-stability is formulated in terms of a family of Lyapunov functions satisfying (45),(46) and with an extra local Lipschitz condition that is uniform in small $T$.

Definition 6. The family $\left(u_{T}, F_{T}\right)$ is said to be equi-globally asymptotically stable (EGAS) by equi-Lipschitz Lyapunov functions if the second statement of Lemma 5 holds and, moreover, for each compact set $\mathcal{X} \subset \mathbb{R}^{n} \backslash\{0\}$ there exist $M>0$ and $T^{*}>0$ such that, for all $x, z \in \mathcal{X}$ and all $\left.T \in\right] 0, T^{*}[$,

$$
\left|V_{T}(x)-V_{T}(z)\right| \leq M|x-z| .
$$

Our first result is expressed in terms of trajectory bounds for $\left(u_{T}, F_{T}^{a}\right)$ and multi-step consistency:

Theorem 3. Let $\beta \in \mathcal{K} \mathcal{L}$ and let $N$ be a bounded neighborhood of the origin. If the family $\left(u_{T}, F_{T}^{a}\right)$ is:

A: multi-step consistent with $\left(u_{T}, F_{T}^{e}\right)$, and

B: $(\beta, N)$-stable,

then

C: the family $\left(u_{T}, F_{T}^{e}\right)$ is $(\beta, N)$-practically stable.

Our second result is expressed in terms of a family of Lyapunov functions for $\left(u_{T}, F_{T}^{a}\right)$ and one-step consistency. It has some relations to the proof technique used to establish the main result of [7]. For simplicity we will only formulate the global result. Nonglobal results and results for stability of sets other than the origin can also be established with the same proof technique. 
Theorem 4. If

A1: $\left(u_{T}, F_{T}^{a}\right)$ is one-step consistent with $\left(u_{T}, F_{T}^{e}\right)$, and

B1: $\left(u_{T}, F_{T}^{a}\right)$ is EGAS by equi-Lipschitz Lyapunov functions

then

C1: there exists $\beta \in \mathcal{K} \mathcal{L}$ such that, for each bounded neighborhood $N$ of the origin, the family $\left(u_{T}, F_{T}^{e}\right)$ is $(\beta, N)$-practically stable.

\subsection{Examples}

Example 4 illustrates Theorem 4 by giving an example where multi-step consistency does not hold but one-step consistency does hold and there is a suitable family of Lyapunov functions. Example 5 shows situation where each element of the family $\left(u_{T}, F_{T}^{a}\right)$ is globally exponentially stable with overshoots uniform in $T$ but where the family $\left(u_{T}, F_{T}^{e}\right)$ fails to be $(\beta, N)$ practically stable for any pair $(\beta, N)$. In reference to Theorem 3 , we use the notation $\overline{\mathbf{C}}$ for this situation.

Example 4. Consider the two-input linear system

$$
\begin{aligned}
& \dot{x}_{1}=x_{1}+u_{1} \\
& \dot{x}_{2}=u_{2}
\end{aligned}
$$

which has exact discretization

$$
\begin{aligned}
& x_{1}(k+1)=e^{T} x_{1}(k)+\left[e^{T}-1\right] u_{1}(k) \\
& x_{2}(k+1)=x_{2}(k)+T u_{2}(k)
\end{aligned}
$$

and Euler approximate discretization

$$
\begin{aligned}
& x_{1}(k+1)=[1+T] x_{1}(k)+T u_{1}(k) \\
& x_{2}(k+1)=x_{2}(k)+T u_{2}(k) .
\end{aligned}
$$

Consider the controller

$$
u(x)=\left\{\begin{array}{l}
{\left[\begin{array}{c}
-2 x_{1} \\
0
\end{array}\right] \text { if } 0<0.1 x_{1}<x_{2}<10 x_{1}} \\
{\left[\begin{array}{c}
-2 x_{1} \\
-x_{2}
\end{array}\right] \text { otherwise. }}
\end{array}\right.
$$

A1, $\overline{\mathbf{A}}$ : It follows from Lemma 2 that $\left(u_{T}, F_{T}^{a}\right)$ is one-step consistent with $\left(u_{T}, F_{T}^{e}\right)$. However, $\left(u_{T}, F_{T}^{a}\right)$ is not multi-step consistent with $\left(u_{T}, F_{T}^{e}\right)$. Indeed, consider the initial condition $\left(\xi_{1}, \xi_{2}\right)=(1,0.1)$. It is easy to see that, in this case, $\left(x_{1}^{a}(k, \xi), x_{2}^{a}(k, \xi)\right)=(1-T)^{k}(1,0.1)$, i.e., the positive 
ray $x_{2}=0.1 x_{1}>0$ is forward invariant for all $T \in(0,1)$. On the other hand, $\left(x_{1}^{e}(1, \xi), x_{2}^{e}(1, \xi)\right)=\left(\left(2-e^{T}\right) 1,(1-T) 0.1\right)$, i.e., for all small $T>0$, $x_{2}^{e}(1, \xi)<10 x_{1}^{e}(1, \xi)$ and $x_{2}^{e}(1, \xi)>0.1 x_{1}^{e}(1, \xi)$ since $e^{T}>1+T$. It follows that, for $k \geq 1, x(k, \xi)$ will take values on the horizontal line given by $x_{2}=(1-T) 0.1$ moving in the direction of decreasing $x_{1}$ until it crosses the positive ray $x_{2}=10 x_{1}$. Let $\bar{k}$ denote the number of steps required to cross the positive ray $x_{2}=10 x_{1}$. It is easy to put an upper and lower bound on $\bar{k} T$ that is independent of $T$. Then since, for all $k \leq \bar{k}$, we have $x_{2}^{e}(k, \xi)=(1-T) 0.1$ while $x_{2}^{a}(k, \xi)=(1-T)^{k} 0.1 \leq e^{-k T} 0.1$, it is clear that the conclusion of Lemma 3 is not satisfied. Hence $\left(u_{T}, F_{T}^{a}\right)$ cannot be multi-step consistent with $\left(u_{T}, F_{T}^{e}\right)$.

B1: We take $V_{T}(x)=\left|x_{1}\right|+\left|x_{2}\right|$. We get, for $T \in(0,1)$ and $0<0.1 x_{1}<$ $x_{2}<10 x_{1}$ :

$$
V_{T}\left(F_{T}^{a}\left(x, u_{T}(x)\right)\right)-V(x)=-T\left|x_{1}\right| \leq-\frac{T}{20}\left[\left|x_{1}\right|+\left|x_{2}\right|\right]
$$

and, otherwise,

$$
V_{T}\left(F_{T}^{a}\left(x, u_{T}(x)\right)\right)-V(x)=-T\left[\left|x_{1}\right|+\left|x_{2}\right|\right] .
$$

It follows that the family $\left(u_{T}, F_{T}^{a}\right)$ is EGAS by equi-Lipschitz Lyapunov functions.

C1: We conclude from Theorem 4 (and also using the homogeneity of $V_{T}(x)$ and $F_{T}^{e}\left(x, u_{T}(x)\right)$ to pass from a semiglobal practical result to a global result and following the steps of the proof of Theorem 4 to get an exponential result) that the family $\left(u_{T}, F_{T}^{e}\right)$ is $\left(\beta, \mathbb{R}^{2}\right)$-stable with $\beta(s, t)$ of the form $k s \exp (-\lambda t)$ with $k>0$ and $\lambda>0$.

Example 5. ( $\mathbf{A}, \overline{\mathbf{B}}, \overline{\mathbf{C}})$ Consider the double integrator, its Euler approximation and its exact discrete-time model:

$$
\text { double integrator: } \begin{aligned}
\dot{x}_{1} & =x_{2} \\
\dot{x}_{2} & =u \\
\text { approximate: } x_{1}(k+1) & =x_{1}(k)+T x_{2}(k) \\
x_{2}(k+1) & =x_{2}(k)+T u(k) \\
\text { exact: } x_{1}(k+1) & =x_{1}(k)+T x_{2}(k)+0.5 T^{2} u(k) \\
x_{2}(k+1) & =x_{2}(k)+T u(k) .
\end{aligned}
$$

The following controller is designed for the Euler model:

$$
u(x)=-\frac{x_{1}}{T}-\frac{2 x_{2}}{T} .
$$

$\overline{\mathbf{C}}:$ The eigenvalues of the exact closed-loop are $\lambda_{1}=1-\frac{T}{2}, \lambda_{2}=-1, \forall T>$ 0 and thus the exact closed-loop model is not $(\beta, N)$-practically stable for any pair $(\beta, N)$. 
A : The eigenvalues of the Euler closed-loop system are $\lambda_{1}=+\sqrt{1-T}, \lambda_{2}=$ $-\sqrt{1-T}$. In a similar way as in the previous example we can show that there exists $b>0$ such that for all $T \in] 0,0.5[$ we have:

$$
|x(k)| \leq b \exp (-0.5 k T)|x(0)|, \forall x(0) \in \mathbb{R}^{2} .
$$

Hence, the approximate closed-loop system is $\left(\beta, \mathbb{R}^{2}\right)$-stable with $\beta(s, t):=$ $b \exp (-0.5 t)$.

$\overline{\mathbf{B}}$ : It now follows from Theorem 3 that $\left(u_{T}, F_{T}^{a}\right)$ is not multi-step consistent with $\left(u_{T}, F_{T}^{e}\right)$. In fact, $\left(u_{T}, F_{T}^{a}\right)$ is not one-step consistent with $\left(u_{T}, F_{T}^{e}\right)$ since

$$
\left|\epsilon_{1}(x)\right|=\left|T^{2} / 2\left(-x_{1} / T-2 x_{2} / T\right)\right|=2 T\left|x_{1}+x_{2}\right|, \forall x \in \mathbb{R}^{2}, \forall T .
$$

\section{Conclusion}

Several recent results on design of sampled-data controllers that appeared in [21,27-31] were overviewed. These results are geared toward providing a unified framework for the digital controller design based either on the continuoustime plant model (Method 1) or on an approximate discrete-time plant model (Method 2). The conditions we presented are easily checkable and the results are applicable to a wide range of plants, controllers and system theoretic properties. Further research is needed to provide control design algorithms based on approximate discrete-time models. Our results on Method 2 provide a unified framework for doing so.

\section{References}

1. J. P. Barbot, S. Monaco, D. Normand-Cyrot and N. Pantalos, Discretization schemes for nonlinear singularly perturbed systems, Proc. CDC'91, pp. 443-448.

2. C. I. Byrnes and W. Lin, "Losslessness, feedback equivalence and the global stabilization of discrete-time systems", IEEE Trans. Automat. Contr., 39 (1994), pp. 83-97.

3. B. Castillo, S. Di Gennaro, S. Monaco and D. Normand-Cyrot, On regulation under sampling, IEEE Trans. Automat. Contr., 42 (1997), pp. 864-868.

4. P. D. Christofides and A. R. Teel, Singular perturbations and input-to-state stability, IEEE Trans. Automat. Contr., 41 (1996), pp. 1645-1650.

5. T. Chen and B. A. Francis, Input-output stability of sampled-data systems, IEEE Trans. Automat. Contr., 36 (1991), pp. 50-58.

6. T. Chen and B. A. Francis, Optimal sampled-data control systems. SpringerVerlag: London, 1995.

7. F. H. Clarke, Y. S. Ledyaev, E. D. Sontag and A. I. Subbotin, Asymptotic controllability implies feedback stabilization, IEEE Trans. Automat. Contr., 42 (1997), pp. 1394-1407.

8. D. Dochain and G. Bastin, Adaptive identification and control algorithms for nonlinear bacterial growth systems, Automatica, 20 (1984), pp. 621-634. 
9. G. F. Franklin, J. D. Powell and M. L. Workman, Digital control of dynamic systems. Addison-Wesley Pub. Co. Inc.: Reading, 1990.

10. B. A. Francis and T. T. Georgiou, Stability theory for linear time-invariant plants with periodic digital controllers, IEEE Trans. Automat. Contr., vol. 33 (1988), pp. 820-832.

11. S. T. Glad, Output dead-beat control for nonlinear systems with one zero at infinity, Systems and Control Letters, 9 (1987), pp. 249-255.

12. G. C. Goodwin, B. McInnis and R. S. Long, Adaptive control algorithm for waste water treatment ad $p H$ neutralization, Optimal Contr. Applic. Meth., 3 (1982), pp. 443-459.

13. L. Grüne, Input-to-state stability of exponentially stabilized semi-linear control systems with inhomogeneous perturbations, preprint (1998).

14. L. Hou, A. N. Michel and H. Ye, Some qualitative properties of sampled-data control systems, IEEE Trans. Automat. Contr., 42 (1997), pp. 1721-1725.

15. P. Iglesias, Input-output stability of sampled-data linear time-varying systems, IEEE Trans. Automat. Contr., 40 (1995), pp. 1646-1650.

16. A. Iserles and G. Söderlind, Global bounds on numerical error for ordinary differential equations, J. Complexity, 9 (1993), pp. 97-112.

17. N. Kazatzis and C. Kravaris, System-theoretic properties of sampled-data representations of nonlinear systems obtained via Taylor-Lie series, Int. J. Control, 67 (1997), pp. 997-1020.

18. H. K. Khalil, Nonlinear systems. Prentice-Hall: New Jersey, 1996.

19. P.E. Kloeden and J. Lorenz. Stable attracting sets in dynamical systems and their one-step discretizations, SIAM J. Num. Anal., 23 (1986), pp. 986-995.

20. B. C. Kuo, Digital control systems. Saunders College Publishing: Ft. Worth, 1992.

21. D. S. Laila and D. Nešić, A note on preservation of dissipation inequalities under sampling: the dynamic feedback case, submitted to Amer. Contr. Conf., 2001.

22. V. Lakshmikantham and S. Leela, Differential and integral inequalities, vol. 1. Academic Press: New York, 1969.

23. I. M. Y. Mareels, H. B. Penfold and R. J. Evans, Controlling nonlinear timevarying systems via Euler approximations, Automatica, 28 (1992), pp. 681-696.

24. S. Monaco and D. Normand-Cyrot, Zero dynamics of sampled nonlinear systems, Syst. Contr. Lett., 11 (1988), pp. 229-234.

25. S. Monaco and D. Normand-Cyrot, Sampling of a linear analytic control system, Proc. CDC'85, pp. 1457-1462.

26. M. S. Mousa, R. K. Miller and A. N. Michel, Stability analysis of hybrid composite dynamical systems: descriptions involving operators and difference equations, IEEE Trans. Automat. Contr., 31 (1986), pp. 603-615.

27. D. Nešić, A. R. Teel and E.D.Sontag, Formulas relating $\mathcal{K} \mathcal{L}$ stability estimates of discrete-time and sampled-data nonlinear systems, Sys. Contr. Lett., 38 (1999), pp. 49-60.

28. D. Nešić, A. R. Teel and P. V Kokotović, Sufficient conditions for stabilization of sampled-data nonlinear systems via discrete-time approximations, Syst. Contr. Lett., 38 (1999), pp. 259-270.

29. D. Nešić and A. R. Teel, Set stabilization of sampled-data differential inclusions via their approximate discrete-time models, to appear in Conf. Decis. Contr., Sydney, 2000. 
30. D. Nešić, D. S. Laila and A. R. Teel, On preservation of dissipation inequalities under sampling, to appear in Conf. Decis. Contr., Sydney, 2000.

31. D. Nešić and P. Dower, Further results on preservation of input-to-state stability under sampling, to appear in ICARV, Singapore, 2000.

32. R. Ortega and D. Taoutaou, A globally stable discrete-time controller for current-fed induction motors, Systems and Control Letters, 28 (1996), pp. 123128.

33. D. H. Owens, Y. Zheng and S. A. Billings, Fast sampling and stability of nonlinear sampled-data systems: Part 1. Existence theorems, IMA J. Math. Contr. Informat., 7 (1990), pp. 1-11.

34. Z. Qu, Robust control of nonlinear uncertain systems. John Wiley \& Sons: New York, 1998.

35. E. D. Sontag, Smooth stabilization implies coprime factorization, IEEE Trans. Automat. Contr., 34 (1989), 435-443.

36. E. D. Sontag, Remarks on stabilization and input-to-state stability, in Proc. CDC, Tampa, USA, 1989.

37. S. A. Svoronos, D. Papageorgiou and C. Tsiligiannis, Discretization of nonlinear control systems via the Carleman linearization, Chemical Eng. Science, 49 (1994), pp. 3263-3267.

38. H. J. Stetter, Analysis of discretization methods for ordinary differential equations. Springer-Verlag: New York, 1973.

39. A. M. Stuart and A. R. Humphries, Dynamical systems and numerical analysis. Cambridge University Press: New York, 1996.

40. A. R. Teel, Connection between Razumikhin-type theorems and the ISS nonlinear small gain theorem, IEEE Trans. Automat. Contr., to appear, 1998.

41. A. R. Teel, D. Nešić and P. V. Kokotović, A note on input-to-state stability of sampled-data nonlinear systems, In Proceedings of the 37th IEEE Conference on Decision and Control, Tampa, Florida, 1998.

42. Y. Zheng, D. H. Owens and S. A. Billings, Fast sampling and stability of nonlinear sampled-data systems: Part 2: Sampling rate estimation, IMA J. Math. Contr. Informat., 7 (1990), pp. 13-33. 